\title{
Estudo da ativação de regiões encefálicas em imagens fMRI
}

\author{
Ricardo Morello Santos ${ }^{1}$, Paulo Sérgio Silva Rodrigues ${ }^{1}$ \\ ${ }^{1}$ Departamento de Ciência da Computação - Centro Universitário FEI \\ São Bernardo do Campo - SP - Brasil \\ \{unifrsantos, psergio\}@fei.edu.br
}

\begin{abstract}
Resumo. Atualmente, $93 \%$ das regiões subcorticais são desconhecidas dos neurocientistas e, portanto, classificadas como terra incognita. Apesar dos recentes avanços nas tecnologias de captura de imagens médicas, a qualidade das imagens coletadas in vivo ainda é baixa em relação às imagens histológicas. Por outro lado, a área de estatística não-extensiva tem avançado e apresentados bons resultados sobretudo em processamento de imagens. Esse trabalho propõe a utilização do filtro sigmoidal baseado em entropia não-extensiva em imagens de ressonância magnética funcional (fMRI), com o intuito de realçar e mapear regiões encefálicas ativadas durante a execução de tarefas específicas.

Abstract. Nowadays, $93 \%$ of subcortical regions are unknown to neuroscientists and, therefore, are classified as terra incognita. Despite the recent advances in medical imaging technologies, the quality of images collected in vivo is still low when compared to the histological images. On the other hand, the area of non-extensive statistics has advanced and presented good results, mainly in image processing. This work proposes the use of the sigmoid fitler with nonextensive entropy in functional magnetic resonance imaging (fMRI), with the purpose of highlighting and mapping brain regions activated during the execution of specific tasks.
\end{abstract}

\section{Introdução e Trabalhos relacionados}

Apesar dos recentes avanços na neurociência, sobretudo no entendimento das regiões encefálicas e suas correlações, ainda restam diversos desafios pendentes. As interações entre regiões corticais e subcorticais, por exemplo, ainda não são completamente compreendidas pelos cientistas [Wager et al. 2008]. Portanto, desconhece-se a origem e motivos de progressão de doenças neurológicas que acometem milhões de indivíduos anualmente.

Até 1940, por exemplo, acreditava-se que surtos de convulsão epiléptica ocorriam por disrupções nas interações convencionais apenas entre regiões corticais. Contudo, atualmente, evidências sugerem a participação de regiões subcorticais em surtos de epilepsia [Norden and Blumenfeld 2002]. Um exemplo de região é o Thalamus, região subcortical responsável, dentre outras coisas, pela regulação do sono. Também, o Thalamus é uma das regiões responsáveis por descargas de espícula ou onda que causam distúrbios de epilepsia durante o sono [Norden and Blumenfeld 2002]. Portanto, o estudo de regiões subcorticais por meio de imagens de ressonância magnética pode auxiliar no diagnóstico preventivo e tratamento de doenças neurológicas.

Entretanto, imagens coletadas in vivo têm qualidade inferior às imagens coletadas em estado post-mortem. Apesar dos recentes avanços em técnicas de imageamento 
e ressonância magnética (MRI), o estudo da terra-incognita, por exemplo, é possível apenas em imagens histológicas [Forstmann et al. 2016]. Alternativas para esse problema incluem melhora nos equipamentos de captura de MRI ou a construção de modelos matemático-computacionais que simulam estruturas e o funcionamento encefálico [Minai 2015]. Por outro lado, uma solução alternativa para este problema envolve o matching de regiões observadas in-vivo com aquelas observáveis em imagens post-mortem.

Porém, esta solução, quando feita de forma manual, depende da análise de centenas de regiões observáveis.A automatização desse processo, por sua vez, depende da melhora nos algoritmos de segmentação, que servem como base para aprimorar a acurácia de algoritmos de detecção de padrões. Técnicas de segmentação clássicas incluem a limiarização por histograma [Pun 1981], que detecta limiares ótimos para separação do espaço de cores de uma imagem com base na distribuição de probabilidade de um histograma, por meio da entropia de Shannon [Shannon and Weaver 1948].

Por sua vez, os trabalhos de Constantino Tsallis na área da estatística deram origem à entropia não-extensiva, uma generalização da entropia convencional de Shannon [Tsallis 1988]. Com base na entropia não-extensiva de Tsallis, novos métodos foram propostos para segmentação e realce de regiões de imagens. Em [Rodrigues and Giraldi 2015], utilizou-se da entropia não-extensiva como função de avaliação de um algoritmo bio-inspirado no comportamento de vagalumes para multisegmentação de imagens médicas. Também, o trabalho de [Gallao and Rodrigues 2015] propôs a generalização da função sigmoide utilizando entropia não-extensiva. Em [Massa et al. 2016], utilizou-se a função sigmoide modificada, denominada $q$-sigmoide, em imagens de câncer mamário para realce das lesões, auxiliando no diagnóstico por sistemas CAD (Computer Aided Diagnosys).

Assim, nesse trabalho, a função $q$-sigmoide será aplicada em imagens MRI de indivíduos durante a execução de uma tarefa, a fim de realçar as regiões ativadas. Este trabalho está organizado da seguinte forma: na Seção 2, será apresentada a teoria por trás da função $q$-simgoide. A seção 3 apresenta a métrica utilizada para encontrar o melhor valor do parâmetro $q$. Na seção 4, serão apresentados os experimentos e tabelas de resultados. Uma conclusão é apresentada na Seção 5.

\section{Metodologia}

A função $q$-sigmoide baseia-se numa variação da função sigmoide tradicional. Considerando uma imagem $I$, com uma região específica com luminância $\beta$ e desvio padrão $\alpha$, a função sigmoide é dada por:

$$
I_{1}(I ; \beta, \alpha, \lambda)=\frac{2}{1+\exp \left(\lambda\left(\frac{|I-\beta|}{\alpha}\right)\right)} .
$$

Assim, regiões dentro do intervalo $\alpha$ do parâmetro $\beta$ assumem valor 1 , enquanto as demais, fora da faixa $[\beta-\alpha, \beta+\alpha]$, são escurecidas. Seguindo a ideia de sistemas estatísticos não-extensivos, a função $q$-sigmoide é uma generalização da função sigmoide tradicional, incorporada para valores de $q=1$. Assim, a função $q$-sigmoide é definida por: 
- $q$-sigmoide para $q<1$ :

$$
\tilde{I}_{1}(I ; \beta, \alpha, \lambda, q)=\frac{2}{1+\left[1+\lambda(1-q)\left(\frac{|I-\beta|}{\alpha}\right)\right]^{\frac{1}{1-q}}},
$$

- $q$-sigmoide para $q>1$ :

$$
\tilde{I}_{2}(I ; \beta, \alpha, \lambda, q)=\left\{\begin{array}{lll}
\frac{1}{1+[1+\lambda(1-q) * F(I)]^{\frac{1}{1-q}}}, & \text { se } \quad I \neq \beta \\
1 & \text { cc }
\end{array}\right.
$$

onde:

$$
F(I)=-\frac{1}{\left(\frac{|I-\beta|}{\alpha}\right)} .
$$

A ideia por trás da utilização da função generalizada da sigmoide é o parâmetro $q$, que permite adaptar a topologia do filtro para cada classe de aplicações, como em [Rodrigues and Giraldi 2011].

\section{Métrica de avaliação para o melhor valor de $q$}

Para definir o melhor parâmetro de $q$ para cada indivíduo, foi utilizada a seguinte medida de similaridade. Considere um slice de um indivíduo, aqui chamado de $I_{s}$. Esse slice tem um mapa de ativação correspondente, $I_{m}$. Esse mapa de ativação, ou máscara, possui um conjunto de regiões com intensidade máxima $I=255$, chamado de $b_{m}$, e um conjunto de regiões com intensidade mínima $I=0$, chamado $p_{m}$. Caso um pixel tenha intensidade $I=255$ no mapa de ativação e no slice correspondente, esse pixel será anexado ao conjunto de correspondências $I=255$ (ou matches), $c_{b}$. Caso um pixel possua intensidade $I=0 \mathrm{em}$ ambas as imagens, ele será acrescentado ao conjunto de correspondências $I=0$, ou $c_{p}$. Por fim, o cálculo do acerto $a_{n}$, para cada slice $n$, será:

$$
a_{n}=\frac{\frac{c_{m}}{b_{m}}+\frac{c_{p}}{p_{m}}}{2}
$$

Para todas as tarefas executadas pelos indivíduos, $n$ sempre será igual a 91 , ou seja, sempre haverá um conjunto de 91 slices para cada uma das sete tarefas executadas pelos 30 indivíduos. Para cada valor de $q$, o erro será somado para cada um dos slices em determinada tarefa, e será calculada a porcentagem de acerto $p_{q}$ :

$$
p_{q}=\frac{\sum_{n=1}^{91} a_{n}}{100}
$$

Caso a porcentagem para o valor atual de $q$ seja superior à maior porcentagem de acerto obtida até então, o novo valor de $q$ é armazenado, bem como sua porcentagem $p_{q}$ correspondente. Assim, para cada indivíduo e cada teste, é definido o valor de $q$ que maximiza a porcentagem de acerto $p_{q}$. 


\section{Experimentos e Resultados}

As imagens foram coletadas da base de dados do Human Connectome Project (HCP). No HCP, estão disponíveis imagens resting-state e task-MRI de 1200 indivíduos, com idades variando entre 22 a 35 anos. Então, para cada indivíduo, foi atribuído um identificador correspondente (aqui chamado de $I D$ ), que pode ser utilizado para encontrá-lo na base de dados do $\mathrm{HCP}^{1}$. As tarefas desempenhadas correspondem a testes emocionais (Emotion), relacionais (Relational), aposta (Gambling), dentre outros. Neste trabalho, estes três testes serão considerados. Sendo assim, para cada teste e cada um dos 1200 indivíduos, um volume MRI está disponível. Para cada volume utilizado, foi feito um processamento para separação em slices.

Neste trabalho, considerou-se como padrão-ouro os mapas de ativação utilizados em [Zuo et al. 2018]. Nele, os autores estudaram as interações e reformulações entre redes de regiões encefálicas quando determinado indivíduo, durante a execução da tarefa resting-state, passa a realizar outra tarefa. Utilizando a base de dados do $\mathrm{HCP}$, os autores adquiriram dados das tarefas 30 indivíduos e identificaram seus mapas de ativação correspondentes utilizando a ferramenta FSL [Jenkinson et al. 2012]. Cada tarefa possui, por sua vez, um mapa de ativação correspondente. Um exemplo de slice separado do indivíduo 101107 durante a execução de uma tarefa Emotion, junto de seu padrão-ouro correspondente, é apresentado na Figura 1.

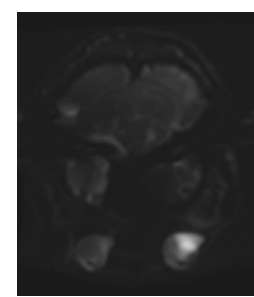

(a)

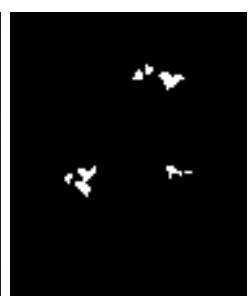

(b)

Figura 1. a) Slice do invidíduo com ID 101107 durante a execução da tarefa de processamento emocional. b) Mapa de ativação correspondente

Para cada teste escolhido, compreendendo Emotion, Gambling e Relational, foi escolhido um mapa de ativação, sendo este correto para o teste ou não, seguido da escolha empírica dos parâmetros, sendo $\beta=0.2, \alpha=0.1 \mathrm{e} l=0.15$. Foram escolhidos testes não correspondentes com o mapa de ativação para verificar se a porcentagem final para cada indivíduo diminui, aumenta ou fica próxima às porcentagens obtidas com o teste correto, para aquele mesmo mapa de ativação.

Após a escolha dos parâmetros, para cada um dos 30 indivíduos, variou-se o parâmetro $q$ de 0.1 a 2, para cada um dos 91 slices. Em cada slice, calculou-se o valor do acerto, que é utilizado no cálculo da porcentagem $p_{q}$ para um dado valor de $q$. Então, verificou-se se a porcentagem é a maior até o momento e, em caso positivo, foram armazenados os valores de $q$ e da porcentagem $p_{q}$. Ao final, foram obtidos os melhores valores de $q$ e $p_{q}$ para cada indivíduo no teste escolhido, de acordo com os parâmetros definidos. Por delimitação de espaço, será exibido um subconjunto de cinco indivíduos para cada tarefa. Os resultados das porcentagens e valores de $q$ otimizados para a tarefa

\footnotetext{
${ }^{1}$ https://db.humanconnectome.org
} 
Emotion são apresentados na Tabela 1. Para a tarefa Gambling, os resultados são mostrados na Tabela 2. Por fim, os resultados para a tarefa Relational são apresentados na Tabela 3.

\begin{tabular}{||ccc||}
\hline Indivíduo & Melhor valor de $q$ & Porcentagem de acerto \\
\hline \hline 101107 & 0.99 & 60.2241 \\
\hline 102008 & 0.8 & 54.2712 \\
\hline 105216 & 0.95 & 56.0232 \\
\hline 113215 & 0.99 & 55.2483 \\
\hline 118730 & 0.98 & 58.9055 \\
\hline
\end{tabular}

Tabela 1. Valores otimizados de $q$ e da porcentagem $p_{q}$ para cada indivíduo no teste de Emotion, utilizando Emotion como mapa de ativação

\begin{tabular}{||ccc||}
\hline Indivíduo & Melhor valor de $q$ & Porcentagem de acerto \\
\hline \hline 101107 & 0.99 & 61.5166 \\
\hline 102008 & 0.91 & 55.2197 \\
\hline 105216 & 0.99 & 52.0868 \\
\hline 113215 & 0.99 & 56.5436 \\
\hline 118730 & 0.99 & 56.7274 \\
\hline
\end{tabular}

Tabela 2. Valores otimizados de $q$ e da porcentagem $p_{q}$ para cada indivíduo no teste de Gambling, utilizando Emotion como mapa de ativação

\begin{tabular}{||ccc||}
\hline Indivíduo & Melhor valor de $q$ & Porcentagem de acerto \\
\hline \hline 101107 & 1.01 & 50.0000 \\
\hline 102008 & 0.75 & 53.7948 \\
\hline 105216 & 0.97 & 56.0395 \\
\hline 113215 & 0.99 & 54.7007 \\
\hline 118730 & 0.91 & 58.6431 \\
\hline
\end{tabular}

Tabela 3. Valores otimizados de $q$ e da porcentagem $p_{q}$ para cada indivíduo no teste de Relational, utilizando Emotion como mapa de ativação

\section{Conclusão}

Nesse trabalho, foi apresentada uma metodologia para realce de regiões encefálicas durante a execução de determinada atividade. Para tanto, foi utilizada a função de ativação sigmoide com kernel entrópico não-extensivo. As imagens do tipo $f M R I$ foram coletadas do HCP, com padrão-ouro sendo os mapas de ativação disponibilizados na literatura. Os testes foram feitos a fim de encontrar o valor de $q$ que possibilita maior combinação com as regiões encontradas no padrão-ouro. Nota-se que, devido ao objetivo de realce de regiões, aquelas regiões utilizadas pelo indivíduo são realçadas, enquanto as demais são escurecidas.

O mapeamento de regiões encefálicas, sobretudo regiões subcorticais, é importante para auxiliar no estudo de origens e tratamentos para diversas doenças neurológicas. Sendo assim, novas técnicas de mapeamento encefálico de imagens in-vivo, que possuem qualidade inferior às imagens histológicas, permitem maior abrangência na identificação 
desses distúrbios. Como trabalhos futuros, pretende-se estudar o uso de algoritmos bioinspirados, que imitam o comportamento da natureza, para segmentação dessas regiões. Assim, pode-se obter resultados próximos do ideal com baixo custo computacional.

\section{Agradecimentos}

Agradecimentos à FAPESP (Fundação de Amparo à Pesquisa do Estado de São Paulo Processo $n^{0}$ 2018/02612-7), CNPq (Conselho Nacional de Desenvolvimento Científico e Tecnológico), CAPES (Coordenação de Aperfeiçoamento de Pessoal de Nível Superior) e ao Centro Universitario FEI.

\section{Referências}

Forstmann, B., Ratcliff, R., and Wagenmakers, E.-J. (2016). Sequential sampling models in cognitive neuroscience: Advantages, applications, and extensions. Annual Review of Psychology, 67(1):641-666. PMID: 26393872.

Gallao, C. and Rodrigues, P. (2015). A q-Gaussian Spatial Filtering. In Proceeding of XI Workshop de Visao Computacional, Uberlandia, MG, Brazil, pages 230-235.

Jenkinson, M., Beckmann, C. F., Behrens, T. E., Woolrich, M. W., and Smith, S. M. (2012). Fsl. NeuroImage, 62(2):782 - 790. 20 YEARS OF fMRI.

Massa, P., Wachs-Lopes, G., Ribeiro, M., and Rodrigues, P. (2016). Realce de Regioes de Cancer Mam?rio Baseado em Fun??es q-Sigmoides. In Proceeding of The 29th SIBGRAPI, Sao Jose dos Campos, SP, Brazil.

Minai, A. A. (2015). Computational Models of Cognitive and Motor Control, pages 665682. Springer Berlin Heidelberg, Berlin, Heidelberg.

Norden, A. D. and Blumenfeld, H. (2002). The role of subcortical structures in human epilepsy. Epilepsy Behavior, 3:219-231.

Pun, T. (1981). Entropic Thresholding: A New Approach. COMPUTER GRAPHICS AND IMAGE PROCESSING, 16(3):210-239.

Rodrigues, P. and Giraldi, G. (2015). Improving a firefly meta-heuristic for multilevel image segmentation using Tsallis entropy. Patterns Analysis and Applications, pages $1-20$.

Rodrigues, P. S. and Giraldi, G. A. (2011). Improving the non-extensive medical image segmentation based on tsallis entropy. Pattern Analysis and Applications, 14(4):369379.

Shannon, C. and Weaver, W. (1948). The Mathematical Theory of Communication. University of Illinois Press, Urbana.

Tsallis, C. (1988). Possible generalization of boltzmann-gibbs statistics. Journal of Statistical Physics, 52:479-487. 10.1007/BF01016429.

Wager, T. D., Davidson, M. L., Hughes, B. L., Lindquist, M. A., and Ochsner, K. N. (2008). Prefrontal-subcortical pathways mediating successful emotion regulation. Neuron, 59:1037-1050.

Zuo, N., Yang, Z., Liu, Y., Li, J., and Jiang, T. (2018). Both activated and less-activated regions identified by functional mri reconfigure to support task executions. In Brain and behavior. 